\title{
Research Article \\ Excellent graphs with respect to domination: subgraphs induced by minimum dominating sets
}

\author{
Vladimir Samodivkin* \\ Department of Mathematics, University of Architecture, Civil Engineering and Geodesy, Sofia 1164, Bulgaria
}

(Received: 12 October 2020. Received in revised form: 4 January 2021. Accepted: 18 January 2021. Published online: 23 January 2021.)

(c) 2021 the author. This is an open access article under the CC BY (International 4.0) license (www.creativecommons.org/licenses/by/4.0/)

\begin{abstract}
A graph $G=(V(G), E(G))$ is $\gamma$-excellent if $V(G)$ is the union of all $\gamma$-sets of $G$, where $\gamma$ stands for the domination number of $G$ and a $\gamma$-set is a dominating set of cardinality $\gamma$. Let $\mathcal{I}$ be a set of all mutually nonisomorphic graphs and let $\emptyset \neq \mathcal{H} \subsetneq \mathcal{I}$. In this paper, the study of the $\mathcal{H}$ - $\gamma$-excellent graphs is initiated. A graph $G$ is $\mathcal{H}$ - $\gamma$-excellent if the following conditions hold: (i) for every $H \in \mathcal{H}$ and for each $x \in V(G)$ there exists an induced subgraph $H_{x}$ of $G$ such that $H$ and $H_{x}$ are isomorphic, $x \in V\left(H_{x}\right)$ and $V\left(H_{x}\right)$ is a subset of some $\gamma$-set of $G$, and (ii) the vertex set of every induced subgraph $H$ of $G$, which is isomorphic to some element of $\mathcal{H}$, is a subset of some $\gamma$-set of $G$. We consider some well-known graphs, including cycles, trees and some cartesian products of two graphs, and for every considered graph we describe its largest set $\mathcal{H} \subsetneq \mathcal{I}$ for which the graph is $\mathcal{H}$ - $\gamma$-excellent. Results on $\gamma$-excellent regular graphs and on a generalized lexicographic product of graphs are presented. Several open problems and questions are also posed.
\end{abstract}

Keywords: domination number; excellent graph; graph product.

2020 Mathematics Subject Classification: 05C69.

\section{Introduction}

All graphs in this paper will be finite, simple, and undirected. We use [8] as a reference for terminology and notation which are not explicitly defined here. In a graph $G=(V(G), E(G))$, for a subset $S \subseteq V(G)$ the subgraph induced by $S$ is the graph $\langle S\rangle$ with vertex set $S$ and two vertices in $\langle S\rangle$ are adjacent if and only if they are adjacent in $G$. The complement $\bar{G}$ of $G$ is the graph whose vertex set is $V(G)$ and two vertices are adjacent in $\bar{G}$ if and only if they are nonadjacent in $G$. The union of two disjoint graphs $G$ and $H$ is denoted by $G \cup H$. For any vertex $x$ of a graph $G, N_{G}(x)$ denotes the set of all neighbors of $x$ in $G, N_{G}[x]=N_{G}(x) \cup\{x\}$ and the degree of $x$ is $\operatorname{deg}_{G}(x)=\left|N_{G}(x)\right|$. The minimum and maximum degrees of a graph $G$ are denoted by $\delta(G)$ and $\Delta(G)$, respectively. For a subset $S \subseteq V(G)$, let $N_{G}[S]=\cup_{v \in S} N_{G}[v]$. Let $X \subseteq V(G)$ and $x \in X$. The $X$-private neighborhood of $x$, denoted by $p n_{G}[x, X]$ or simply by $p n[x, X]$ (if the graph is clear from the context), is the set $\{y \in V(G) \mid N[y] \cap X=\{x\}\}$. A leaf is a vertex of degree one and a support vertex is a vertex adjacent to a leaf. A vertex which separates two other vertices of the same component is a cut-vertex, and an edge separating its ends is a bridge. The distance dist ${ }_{G}(x, y)$ in $G$ of two vertices $x, y$ is the length of a shortest $x-y$ path in $G$; if no such path exists, we set $\operatorname{dist}_{G}(x, y):=\infty$. An isomorphism of two graphs $G$ and $H$ is a bijection $f: V(G) \rightarrow V(H)$ between the vertex sets of $G$ and $H$ such that any two vertices $u$ and $v$ of $G$ are adjacent in $G$ if and only if $f(u)$ and $f(v)$ are adjacent in $H$. If an isomorphism exists between two graphs, then the graphs are called isomorphic and denoted as $G \simeq H$. We write $K_{n}$ for the complete graph of order $n$ and $P_{n}$ for the path on $n$ vertices. Let $C_{m}$ denote the cycle of length $m$. A complete $r$-partite graph $K_{n_{1}, n_{2}, \ldots, n_{r}}$ is a graph whose vertex set can be partitioned into $r$ parts, say $V_{1}, V_{2}, \ldots, V_{r}$, such that (a) no two vertices within the same part are adjacent, (b) there is an edge between every two vertices of different parts of the partition, and (c) $\left|V_{i}\right|=n_{i}, i=1,2, \ldots, r$. The 1-corona, denoted $\operatorname{cor}(U)$, of a graph $U$ is the graph obtained from $U$ by adding a degree-one neighbor to every vertex of $U$. We use the notation $[k]$ for $\{1,2, . ., k\}$.

An independent set is a set of vertices in a graph, no two of which are adjacent. The independence number of $G$, denoted $\beta_{0}(G)$, is the maximum size of an independent set in $G$. A subset $D \subseteq V(G)$ is called a dominating set (or a total dominating set) in $G$, if for each $x \in V(G)-D$ (or for each $x \in V(G)$, respectively) there exists a vertex $y \in D$ adjacent to $x$. A dominating set $R$ of a graph $G$ is a restrained dominating set (or an outer-connected dominating set) in $G$, if every vertex in $V(G)-R$ is adjacent to a vertex in $V(G)-R$ (or $V(G)-R$ induces a connected graph, respectively). The minimum number of vertices of a dominating set in a graph $G$ is the domination number $\gamma(G)$ of $G$. Analogously the total domination number $\gamma_{t}(G)$, the restrained domination number $\gamma_{r}(G)$ and the outer-connected domination number $\gamma^{o c}(G)$ are defined. The minimum 
cardinality of a set $S$ which is simultaneously total dominating and restrained dominating in $G$ is called the total restrained domination number $\gamma_{t r}(G)$ of $G$. The minimum cardinality of a set $S$ which is simultaneously total dominating and outerconnected dominating in $G$ is called the total outer-connected domination number $\gamma_{t}^{o c}(G)$ of $G$. The independent domination number of $G$, denoted by $i(G)$, is the minimum size of an independent dominating set.

For a graph $G$, let $\pi$ be a graphical property that can be possessed, or satisfied by the subsets of $V$. For example, being a maximal complete subgraph, a maximal independent set, acyclic, a closed/open neighborhood, a minimal dominating set, etc. Suppose that $f_{\pi}$ and $F_{\pi}$ are the associated graph invariants: the minimum and maximum cardinalities of a set with property $\pi$. Let $\mu \in\left\{f_{\pi}, F_{\pi}\right\}$. For a graph $G$, denote by $\mathrm{M}_{\mu}(G)$ the family of all subsets of $V(G)$ each of which has property $\pi$ and cardinality $\mu(G)$. Each element of $\mathrm{M}_{\mu}(G)$ is called a $\mu$-set of $G$. Fricke et al. [6] define a graph $G$ to be $\mu$-excellent if each its vertex belongs to some $\mu$-set. Perhaps historically the first results on $\mu$-excellent graphs were published by Berge [1] who introduced the class of $B$-graphs consisting of all graphs in which every vertex is in a maximum independent set. Of course all $B$-graphs form the class of $\beta_{0}$-excellent graphs. The study of excellent graphs with respect to the some domination related parameters was initiated by Fricke et al. [6] and continued e.g. in [3,9,10,14,18,20,23].

In this paper we focus on the following subclass of the class of $\mu$-excellent graphs.

Definition 1.1. Let $\mathcal{I}$ be a set of all mutually nonisomorphic graphs and $\emptyset \neq \mathcal{H} \subsetneq \mathcal{I}$. We say that a graph $G$ is $\mathcal{H}$ - $\mu$-excellent if the following hold:

(i) For each $H \in \mathcal{H}$ and for each $x \in V(G)$ there exists an induced subgraph $H_{x}$ of $G$ such that $H$ and $H_{x}$ are isomorphic, $x \in V\left(H_{x}\right)$ and $V\left(H_{x}\right)$ is a subset of some $\mu$-set of $G$.

(ii) For each induced subgraph $H$ of $G$, which is isomorphic to some element of $\mathcal{H}$, there is a $\mu$-set of $G$ having $V(H)$ as a subset.

By the above definition it immediately follows that each $\mathcal{H}$ - $\mu$-excellent graph is $\mu$-excellent. If a graph $G$ is $\mathcal{H}$ - $\mu$-excellent and $\mathcal{H}$ contains only one element, e.g. $\mathcal{H}=\{H\}$, we sometimes omit the brackets and say that a graph $G$ is $H$ - $\mu$-excellent. Define the $\mu$-excellent family of induced subgraphs of a $\mu$-excellent graph $G$, denoted by $G\langle\mu\rangle$, as the family of all graphs $H \in \mathcal{I}$ for which $G$ is $H$ - $\mu$-excellent. The next two observations are obvious.

Observation 1.1. If $G$ is a $\mu$-excellent graph, then $\left\{K_{1}\right\} \subseteq G\langle\mu\rangle$ and $\mu(G) \geq \max \{|V(H)| \mid H \in G\langle\mu\rangle\}$.

Observation 1.2. Let a graph $G$ be both $\mu$-excellent and $\nu$-excellent. If the set of all $\mu$-sets and the set of all $\nu$-sets of $G$ coincide, then $G\langle\mu\rangle=G\langle\nu\rangle$.

As first examples of $\mathcal{H}$ - $\mu$-excellent graphs let us consider the case $\mu=\beta_{0}$. Clearly, any $\beta_{0}$-excellent graph $G$ is $\left\{\overline{K_{1}}, \overline{K_{\beta_{0}(G)}}\right\}$ $\beta_{0}$-excellent. A graph is r-extendable if every independent set of size $r$ is contained in a maximum independent set (Dean and Zito [4]). Clearly, a graph is $\left\{\overline{K_{1}}, \overline{K_{2}}, . ., \overline{K_{r}}\right\}-\beta_{0}$-excellent if and only if it is $s$-extendable for all $s=1,2, . ., r$. Plummer [15] define a graph $G$ to be well covered whenever $G$ is $k$-extendable for every integer $k$. In other words, a graph $G$ is well covered if and only if $G\left\langle\beta_{0}\right\rangle=\left\{\overline{K_{1}}, \overline{K_{2}}, . ., \overline{K_{\beta_{0}(G)}}\right\}$.

In this paper we concentrate mainly on excellent graphs with respect to the domination number $\gamma$. We give basic terminologies and notations in the rest of this section. In Section 2 we describe the $\gamma$-excellent family of induced subgraphs for some well known graphs. In Section 3 we show that, under appropriate restrictions, the generalized lexicographic product of graphs has the same excellent family of induced subgraphs with respect to six domination-related parameters. Section 4 contains results on $\gamma$-excellent regular graphs and trees. We conclude in Section 5 with some open problems.

\section{Examples}

Here we find the $\gamma$-excellent family of induced subgraphs of some well known graphs.

Example 2.1. Let $G$ be a connected graph with $\gamma(G)=2$. In [11] it is proved that (in our terminology) $G$ is $K_{2}$ - $\gamma$-excellent if and only if $G$ is a complete r-partite graph $K_{n_{1}, n_{2}, \ldots, n_{r}}, n_{i} \geq 2, i=1,2, \ldots, r \geq 2$. Clearly $K_{2,2, \ldots, 2}\langle\gamma\rangle=\left\{K_{1}, K_{2}, \overline{K_{2}}\right\}$ and $K_{n_{1}, n_{2}, \ldots, n_{r}}\langle\gamma\rangle=\left\{K_{1}, K_{2}\right\}$ when $n_{s} \geq 3$ for some $s \in[r]$.

Example 2.2. Let $\nu \in\{\gamma, i\}$. Then all the following hold:

(i) (folklore) $\nu\left(P_{n}\right)=\lceil n / 3\rceil$ and $\nu\left(C_{r}\right)=\lceil r / 3\rceil . C_{r}$ is $\nu$-excelent for all $r \geq 3 . P_{n}$ is $\nu$-excellent if and only if $n=2$ or $n \equiv 1$ $(\bmod 3)$.

(ii) $P_{n}\langle\nu\rangle=\left\{K_{1}\right\}$ when $n \in\{1,2\} \cup\{7,10, \ldots\}$ and $P_{4}\langle\nu\rangle=\left\{K_{1}, \overline{K_{2}}\right\}$ 
(iii) $C_{5}\langle\nu\rangle=\left\{K_{1}, \overline{K_{2}}\right\}$ and $C_{3 r}\langle\nu\rangle=C_{5+3 r}\langle\nu\rangle=\left\{K_{1}\right\}, r \geq 1$.

(iv) $C_{7}\langle\gamma\rangle=\left\{K_{1}, K_{2}, \overline{K_{2}}, \overline{K_{3}}\right\}$, and $C_{3 r+1}\langle\gamma\rangle=\left\{K_{1}, K_{2}, \overline{K_{2}}\right\}$ for $r \neq 2$.

(v) $C_{7}\langle i\rangle=\left\{K_{1}, \overline{K_{2}}, \overline{K_{3}}\right\}$ and $C_{3 r+1}\langle i\rangle=\left\{K_{1}, \overline{K_{2}}\right\}$ for $r \neq 2$.

The proof is straightforward and hence we omit it.

Denote by (CEA) the class of all graphs $G$ such that $\gamma(G+e) \neq \gamma(G)$ for all $e \in E(\bar{G})$.

Example 2.3. Let a noncomplete graph $G$ be in (CEA). It is well known fact that any two nonadjacent vertices of $G$ belong to some $\gamma$-set of $G$ (Sumner and Blitch [21]). In other words, $G$ is $\left\{K_{1}, \overline{K_{2}}\right\}$ - $\gamma$-excellent graph.

Proposition 2.1. Let $G$ be a graph with $\beta_{0}(G)=\gamma(G)=s$. Then $G$ is $\left\{\overline{K_{1}}, \overline{K_{2}}, \ldots, \overline{K_{s}}\right\}$ - $\gamma$-excellent and $G\langle i\rangle=G\left\langle\beta_{0}\right\rangle=$ $\left\{\overline{K_{1}}, \ldots, \overline{K_{s}}\right\}$ (for the second conclusion, see [15]).

Proof. Every independent set of $G$ is a subset of a maximal independent set. Since each maximal independent set is always a dominating set and $\beta_{0}(G)=\gamma(G)=s$, the result immediately follows.

The Cartesian product of two graphs $G$ and $H$ is the graph $G \square H$ whose vertex set is the Cartesian product of the sets $V(G)$ and $V(H)$. Two vertices $\left(u_{1}, v_{1}\right)$ and $\left(u_{2}, v_{2}\right)$ are adjacent in $G \square H$ precisely when either $u_{1}=u_{2}$ and $v_{1} v_{2} \in E(H)$ or $v_{1}=v_{2}$ and $u_{1} u_{2} \in E(G)$. It is clear from this definition that $G \square H \simeq H \square G$ and if $G$ or $H$ is not connected then $G \square H$ is not connected.

Example 2.4. Let $G=K_{m} \square K_{n}, n \geq m \geq 2$. Then $G\langle i\rangle=G\left\langle\beta_{0}\right\rangle=\left\{\overline{K_{1}}, \ldots, \overline{K_{m}}\right\}$. If $n>m$, then $G\langle\gamma\rangle=\left\{\overline{K_{1}}, \ldots, \overline{K_{m}}\right\}$. If $n=m$, then $G\langle\gamma\rangle=\left\{\overline{K_{1}}, \ldots, \overline{K_{m}}\right\} \cup\left\{K_{1}, K_{2}, \ldots, K_{m}\right\} \cup\left\{K_{p} \cup \overline{K_{q}} \mid(p \geq 2) \wedge(q \geq 1) \wedge(p+q \leq m)\right\}$.

Proof. Let $G=K_{m} \square K_{n}, n \geq m \geq 2$. We consider $G$ as an $m \times n$ array of vertices $\left\{x_{i, j} \mid(1 \leq i \leq m) \wedge(1 \leq j \leq n)\right\}$, where the closed neighborhood of $x_{i, j}$ is the union of the sets $A_{i}=\left\{x_{i, 1}, x_{i, 2}, \ldots, x_{i, n}\right\}$ and $B_{j}=\left\{x_{1, j}, x_{2, j}, \ldots, x_{m, j}\right\}$. Then $\left\langle A_{i}\right\rangle \simeq K_{n}$ and $\left\langle B_{j}\right\rangle \simeq K_{m}$. It is well-known that [7] (a) $\gamma(G)=i(G)=\beta_{0}(G)=m$, (b) $A_{1}, A_{2}, \ldots, A_{m}$ are $\gamma$-sets of $G$, and if $m=n, B_{1}, B_{2}, \ldots, B_{n}$ are also $\gamma$-sets of $G$. Hence, by Proposition $2.1, G$ is $\left\{\overline{K_{1}}, \overline{K_{2}}, \ldots, \overline{K_{m}}\right\}$ - $\gamma$-excellent and $G\langle i\rangle=G\left\langle\beta_{0}\right\rangle=$ $\left\{\overline{K_{1}}, \ldots, \overline{K_{m}}\right\}$. Suppose that $G$ is $H$ - $\gamma$-excellent. Then there is a $\gamma$-set $D$ of $G$ such that $\langle D\rangle$ has an induced subgraph $H_{1} \simeq H$. Assume that $H$ has at least one edge.

Case 1: $m<n$. Clearly $\left|A_{i} \cap D\right|=1$ for all $i=1,2, . ., m$. Because of symmetry, we assume without loss of generality that $D \cap B_{j}$ is empty for all $j>m$. Define now the set $D^{t}=\left\{x_{r, s} \mid x_{s, r} \in D\right\}$. Since $H$ is not edgeless, $\left|D \cap B_{j}\right|>1$ for some $j \leq m$. But then $\left|D^{t} \cap A_{j}\right|>1$, which means that $D^{t}$ is not a $\gamma$-set of $G$. Since $\langle D\rangle \simeq\left\langle D^{t}\right\rangle, G$ is not $H$ - $\gamma$-excellent. Thus, $G\langle\gamma\rangle=\left\{\overline{K_{1}}, \ldots, \overline{K_{s}}\right\}$.

Case 2: $m=n$. Obviously in this case exactly one of $\left|A_{i} \cap D\right|=1$ for all $i=1,2, . ., m$ and $\left|B_{j} \cap D\right|=1$ for all $j=1,2, \ldots, m$ holds. Say the first is valid. Let $R_{1}$ be a $l$-order component of $\langle H\rangle$ for some $l \geq 2$. For the sake of symmetry, we can assume that all elements of $R_{1}$ are in $B_{1}$ and $D \subset \cup_{s=1}^{p} B_{s}$, where $D \cap B_{s}$ is not empty for all $s \in[p]$. Clearly $p \leq m-l+1$. Suppose that $\langle D\rangle$ has another nontrivial component. Then the difference $m-p$ is not less than $l$. Define the set $D_{1}=$ $\left(D-V\left(R_{1}\right)\right) \cup\left\{x_{1, p+1}, x_{1, p+2}, \ldots, x_{1, p+l}\right\}$. Clearly $D_{1}$ is not a $\gamma$-set of $G$ and $\left\langle D_{1}\right\rangle \simeq\langle D\rangle$. Thus $R_{1}$ is the only nontrivial component of $\langle D\rangle$. Hence $H$ is either a complete graph or a union of complete and edgeless graph. Finally, it is easy to see that for each such a graph $H, G$ is $H$ - $\gamma$-excellent.

We need the following negative result.

Theorem 2.1. There is no $P_{3}$ - $\gamma$-excellent graph $G$ with $\gamma(G)=3$.

Proof. Assume that $G$ is a $P_{3}$ - $\gamma$-excellent graph, $\gamma(G)=3$ and $x_{1}, x_{2}, x_{3}$ is an induced path in $G$. Since $X=\left\{x_{1}, x_{2}, x_{3}\right\}$ is a $\gamma$-set of $G$, there is $y_{i} \in p n\left[x_{i}, X\right], i=1,2,3$. Then $\left\{x_{1}, x_{2}, y_{2}\right\}$ is a $\gamma$-set of $G$, which implies $y_{2} y_{3} \in E(G)$. But now no vertex of the induced path $y_{2}, y_{3}, x_{3}$ is adjacent to $x_{1}$, a contradiction.

Example 2.5. $\overline{K_{3} \square K_{n}}\langle\gamma\rangle=\left\{K_{1}, K_{2}, \overline{K_{2}}, K_{1} \cup K_{2}, \overline{K_{3}}, K_{3}\right\}$ when $n \geq 3$, and $\overline{K_{p} \square K_{n}}\langle\gamma\rangle=\left\{K_{1}, K_{2}, \overline{K_{2}}, K_{1} \cup K_{2}, K_{3}\right\}$ when $n \geq p \geq 4$.

Proof. First note that $\overline{K_{3} \square K_{3}} \simeq K_{3} \square K_{3}$ and by Example 2.4 it immediately follows that $\overline{K_{3} \square K_{3}}\langle\gamma\rangle=\left\{K_{1}, K_{2}, \overline{K_{2}}, K_{1} \cup\right.$ $\left.K_{2}, \overline{K_{3}}, K_{3}\right\}$. So, let $n \geq 4$ and $m$ be an integer such that $n \geq m \geq 3$. It is well known that $[7] \gamma\left(\overline{K_{m} \square K_{n}}\right)=3 \leq m=$ $i\left(\overline{K_{m} \square K_{n}}\right)$. Let us consider the graph $G_{m, n}=\overline{K_{m} \square K_{n}}$ as a $m \times n$ array of vertices $\left\{a_{i, j} \mid(1 \leq i \leq m) \wedge(1 \leq j \leq n)\right\}$, with an adjacency $N\left(a_{i, j}\right)=V\left(G_{m, n}\right)-\left(Y_{i} \cup Z_{j}\right)$, where $Y_{i}=\cup_{k=1}^{n}\left\{a_{i, k}\right\}$ and $Z_{j}=\cup_{r=1}^{m}\left\{a_{r, j}\right\}$. Remark now that: 
(a) $\left\langle\left\{a_{i, j}, a_{k, l}, a_{r, s}\right\}\right\rangle \simeq K_{3}$ if and only if both 3-tuples $(i, k, r)$ and $(j, l, s)$ consist of paired distinct integers. The vertices of each triangle of $G_{m, n}$ form a $\gamma$-set. Every two adjacent vertices $a_{i, j}$ and $a_{k, l}$ belong to a triangle.

(b) All induced subgraphs isomorphic to $K_{1} \cup K_{2}$ are $\left\langle\left\{a_{i, j}, a_{k, l}, a_{i, l}\right\}\right\rangle$ and $\left\langle\left\{a_{i, j}, a_{k, l}, a_{k, j}\right\}\right\rangle$, where $i \neq k$ and $j \neq l$. The vertices of each such a subgraph form a $\gamma$-set. Every two vertices belong to an induced subgraph isomorphic to $K_{1} \cup K_{2}$.

(c) Each 3-cardinality subset of $Z_{j}$ is independent and it is not dominating.

Theorem 2.1 together with (a)-(c) immediately lead to the required.

To continue we need the following theorem and definitions.

Theorem 2.2. [5] $\gamma(G \square H) \geq \min \{|V(G)|,|V(H)|\}$ for any two arbitrary graphs $G$ and $H$.

A $G$-layer of the Cartesian product $G \square H$ is the set $\{(u, y) \mid u \in V(G)\}$, where $y \in V(H)$. Analogously an $H$-layer is the set $\{(x, v) \mid v \in V(H)\}$, where $x \in V(G)$. A subgraph of $G \square H$ induced by a $G$-layer or an $H$-layer is isomorphic to $G$ or $H$, respectively.

Theorem 2.3. Let $H$ be a connected noncomplete $n$-order graph and $p \geq n \geq 3$. If each induced subgraph of $K_{p} \square H$ which is isomorphic to $H$ has as a vertex set some $H$-layer, then $\gamma\left(K_{p} \square H\right)=n$ and $K_{p} \square H$ is a $H$ - $\gamma$-excellent graph.

Proof. Each $H$-layer of $K_{p} \square H$ is a dominating set of $K_{p} \square H$. Hence $\gamma\left(K_{p} \square H\right) \leq|V(H)|=n$. Since $p \geq n$, by Theorem 2.2 we have that each $H$-layer is a $\gamma$-set of $K_{p} \square H$. It remains to note that clearly each vertex of $K_{p} \square H$ belongs to some $H$-layer.

The next example serves as an illustration of the above theorem.

Example 2.6. If $p \geq n \geq 5$, then the graph $K_{p} \square C_{n}$ is $C_{n}$ - $\gamma$-excellent.

Proof. Let $H$ be an induced subgraph of $K_{p} \square C_{n}$ which is isomorphic to $C_{r}$. It is easy to see that if the vertex set of $H$ is not a $C_{n}$-layer, then either $r \in\{3,4\}$ or $r \geq n+2$. The required immediately follows by Theorem 2.3.

\section{Generalized lexicographic product}

Let $G$ be a graph with vertex set $V(G)=\{\mathbf{1}, \mathbf{2}, \ldots, \mathbf{n}\}$ and let $\Phi=\left(F_{1}, F_{2}, \ldots, F_{n}\right)$ be an ordered $n$-tuple of paired disjoint graphs. Denote by $G[\Phi]$ the graph with vertex set $\cup_{i=1}^{n} V\left(F_{i}\right)$ and edge set defined as follows: (a) $F_{1}, F_{2}, \ldots, F_{n}$ are induced subgraphs of $G[\Phi]$, and (b) if $x \in V\left(F_{i}\right), y \in V\left(F_{j}\right), i, j \in[n]$ and $i \neq j$, then $x y \in E(G[\Phi])$ if and only if $\mathbf{i j} \in E(G)$. A graph $G[\Phi]$ is called the generalized lexicographic product of $G$ and $\Phi$. If $F_{i} \simeq F$ for every $i=1,2, \ldots, n$, then $G[\Phi]$ becomes the standard lexicographic product $G[F]$. Each subset $U=\left\{u_{1}, u_{2}, \ldots, u_{n}\right\} \subseteq V(G[\Phi])$ such that $u_{i} \in V\left(F_{i}\right)$, for every $i \in[n]$, is called a $G$-layer. From the definition of $G[\Phi]$ it immediately follow:

(A) (folklore) $G[\Phi] \simeq G$ if and only if $G[\Phi]=G\left[K_{1}\right] . G[F] \simeq F$ if and only if $G \simeq K_{1}$. If $G$ has at least two vertices, then $G[\Phi]$ is connected if and only if $G$ is connected. If $G$ is edgeless, then $G[\Phi]=\cup_{i=1}^{n} F_{i}$. For any $G$-layer $U=\left\{u_{1}, u_{2}, \ldots, u_{n}\right\}$ the bijection $f: V(G) \rightarrow U$ defined by $f(\mathbf{i})=u_{i} \in V\left(F_{i}\right)$ is an isomorphism between $G$ and $\langle U\rangle$. For any $x \in V\left(F_{i}\right)$ and $y \in V\left(F_{j}\right), i \neq j$, is fulfilled $\operatorname{dist}_{G[\Phi]}(x, y)=\operatorname{dist}_{G}(\mathbf{i}, \mathbf{j})$.

The equality $\operatorname{dist}_{G[\Phi]}(x, y)=\operatorname{dist}_{G}(\mathbf{i}, \mathbf{j})$ will be used in the sequel without specific references.

Theorem 3.1. Given a graph $G[\Phi]$, where $G$ is connected of order $n \geq 2$ and $\left|V\left(F_{k}\right)\right| \geq 3$ for all $k \in[n]$. Then $G[\Phi]\langle\gamma\rangle=$ $G[\Phi]\left\langle\gamma_{r}\right\rangle=G[\Phi]\left\langle\gamma^{o c}\right\rangle$ and $G[\Phi]\left\langle\gamma_{t}\right\rangle=G[\Phi]\left\langle\gamma_{t r}\right\rangle=G[\Phi]\left\langle\gamma_{t}^{o c}\right\rangle$. If $\gamma\left(F_{k}\right) \geq 3$ for all $k \in[n]$, then $G[\Phi]\langle\gamma\rangle=G[\Phi]\left\langle\gamma_{r}\right\rangle=$ $G[\Phi]\left\langle\gamma^{o c}\right\rangle=G[\Phi]\left\langle\gamma_{t}\right\rangle=G[\Phi]\left\langle\gamma_{t r}\right\rangle=G[\Phi]\left\langle\gamma_{t}^{o c}\right\rangle$.

Proof. Let $\mu \in\left\{\gamma, \gamma_{t}\right\}$ and $D$ a $\mu$-set of $G[\Phi]$. Assume there is $i \in[n]$ such that $V\left(F_{i}\right) \cap D=\left\{v_{1}, v_{2}, \ldots, v_{r}\right\}$, where $r \geq 2$. Then clearly for each $\mathbf{j} \in N(\mathbf{i}), V\left(F_{j}\right) \cap D$ is empty and for any $u_{j} \in V\left(F_{j}\right)$ the set $\left(D-\left\{v_{2}, \ldots, v_{r}\right\}\right) \cup\left\{u_{j}\right\}$ is a dominating set of $G[\Phi]$ or a total dominating set of $G[\Phi]$ depending on whether $\mu=\gamma$ or $\mu=\gamma_{t}$, respectively. Hence $r=2$. Since $G$ is connected of order $n \geq 2$ and $\left|V\left(F_{i}\right)\right| \geq 3$ for all $i \in[n]$, the graph $\langle V(G[\Phi])-D\rangle$ is connected. Therefore the first two equality chains are correct.

Finally, let $D_{1}$ be a $\gamma$-set of $G[\Phi]$ and $\gamma\left(F_{k}\right) \geq 3$ for all $k \in[n]$. Then clearly for every $i \in[n]$ the sets $D$ and $V\left(F_{i}\right)$ must have no more than one element in common. But this immediately implies that $D_{1}$ is a total dominating set of $G[\Phi]$. Thus, the last equality chain holds. 
Theorem 3.2. Given a graph $G[\Phi]$, where $G$ is connected of order $n \geq 2$ and $F_{k}$ is complete with $\left|V\left(F_{k}\right)\right| \geq 2$ for all $k \in[n]$. Then $G[\Phi]$ is $\overline{K_{s}}-\gamma$-excellent if and only if $G$ is $\overline{K_{s}}-\gamma$-excellent.

Proof. Recall that any $G$-layer of $G[\Phi]$ induces a graph isomorphic to $G$. We need the following claim.

Claim 1. (i) Each $\gamma$-set $D$ of $G[\Phi]$ is contained in a $G$-layer of $G[\Phi]$; moreover, $D$ is a $\gamma$-set of each subgraph of $G[\Phi]$ that is induced by a $G$-layer containing $D$. (ii) If $D^{*}$ is a $\gamma$-set of some subgraph of $G[\Phi]$ that is induced by a $G$-layer, then $D^{*}$ is a $\gamma$-set of $G[\Phi]$.

Proof of Claim 1. If $D$ is a $\gamma$-set of $G[\Phi]$, then since all $F_{i}$ 's are complete $\left|D \cap V\left(F_{i}\right)\right| \leq 1$ for all $i \in[n]$. But then $D$ is a dominating set of any subgraph of $G[\Phi]$ that is induced by a $G$-layer containing $D$. In particular this leads to $\gamma(G[\Phi]) \leq \gamma(G)$.

If $D^{*}$ is a $\gamma$-set of some subgraph of $G[\Phi]$ that is induced by a $G$-layer, then again by the fact that all $F_{i}$ 's are complete, it follows that $D^{*}$ is a dominating set of $G[\Phi]$. This clearly leads to $\gamma(G[\Phi]) \geq \gamma(G)$.

Thus $\gamma(G[\Phi])=\gamma(G)$ implying the required.

$\Leftarrow$ Choose $u \in V(G[\Phi])$ arbitrarily. Then there is a $G$-layer $U$ containing $u$. Since $G$ is $\overline{K_{s}}-\gamma$-excellent, there is a $\gamma$-set $D^{*}$ of $\langle U\rangle$ that contains $s$ paired nonadjacent vertices one of which is $u$. By Claim $1, D^{*}$ is a $\gamma$-set of $G[\Phi]$.

If $R$ is a $s$-vertex independent set in $G[\Phi]$, then since all $F_{i}$ 's are complete graphs, $R$ is a subset of some $G$-layer. The rest is as above.

$\Rightarrow$ Let $L=\left\{l_{1}, l_{2}, \ldots, l_{n}\right\}$ be a $G$-layer of $G[\Phi]$, where $l_{i} \in V\left(F_{i}\right), i \in[n]$. Choose $l_{r} \in L$ arbitrarily. Since $G[\Phi]$ is $\overline{K_{s}}-\gamma$ excellent, there is an $s$-vertex independent set $I_{s}$ of $G[\Phi]$ and a $\gamma$-set $D$ of $G[\Phi]$ such that $u \in I_{s} \subseteq D$. By Claim $1, D$ is a $\gamma$-set of some subgraph induced by a $G$-layer of $G[\Phi]$. Since all $F_{i}$ 's are complete, without loss of generality, we can assume that $D \subseteq L$.

Let $R$ be a $s$-vertex independent set of $L$. Then there is a $\gamma$-set $D_{1}$ of $G[\Phi]$ which has $R$ as a subset. By Claim $1 D_{1}$ is a $\gamma$-set of a graph induced by some $G$-layer and as above we can assume that $D_{1} \subseteq L$.

\section{Regular graphs and trees}

To present the next results on regular graphs, we need the following theorem.

Theorem 4.1. Let $G$ be a n-order graph with minimum degree $\delta$. Then $\gamma(G) \leq n \delta /(3 \delta-1)$ when $\delta \in\{3,4,5\}$ (see [16], [19] and [22], respectively).

For any 5-regular graph $G$ with $\gamma(G)=3$, the bound stated in Theorem 4.1 can be improved by 3 .

Proposition 4.1. Let $G$ be a 5-regular graph with $\gamma(G)=3$. Then $n \geq 12$.

Proof. By Theorem 4.1 we have $n \geq 9$. Since there is no 5-regular graphs of odd order, $n \geq 10$ is even. Note that there are exactly sixty 5 -regular graphs of order $10[12,13]$. Their adjacency lists can be found in [13]. A simple verification shows that each of these graphs has the domination number equals to 2 .
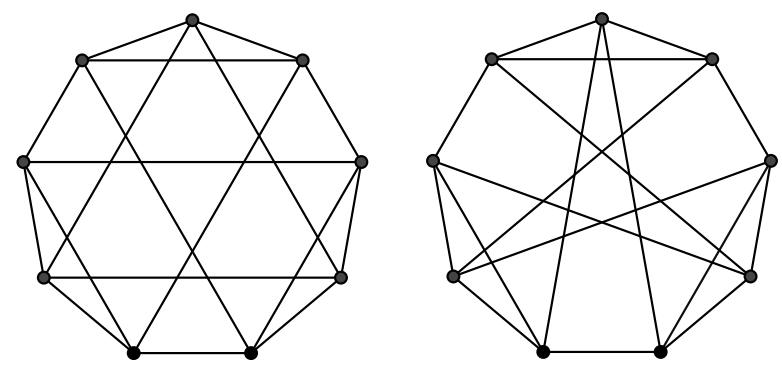

Figure 1: The two 4-regular $K_{3}$ - $\gamma$-excellent graphs of order 9. The graph on the right is $K_{3} \square K_{3}$.

Theorem 4.2. Let $G$ be a s-regular $K_{r}$ - $\gamma$-excellent $n$-order connected graph with $\gamma(G)=r$, where $n>s \geq r \geq 3$. Then the following assertions hold.

(i) $n \leq r(s-r+2)$.

(ii) If $r=3$, then $s \geq 4$ with equality if and only if $n=9$ and $G$ is one of the graphs depicted in Fig.1.

(iii) If $r=3$ and $s=5$, then $n=12$. 
Proof. (i) Let $H \simeq K_{r}$ be a subgraph of $G$. Each vertex of $H$ is adjacent to $s-r+1$ vertices outside $V(H)$. Hence $n \leq r+r(s-r+1)=r(s-r+2)$.

(ii) Since $r=3$, we have $\gamma(G)=3$ and $n \leq 3 s-3$. By Theorem 4.1 we obtain $8 \leq n$ when $s=3$ and $9 \leq n$ when $s \geq 4$. Thus $s \geq 4$ and if the equality holds, then $n=9$. There are exactly 164 -regular graphs of order 9 [13]. An immediate verification shows that among them only the graphs depicted in Fig. 1 are $K_{3}$ - $\gamma$-excellent.

(iii) By (i), $n \leq 12$ and by Proposition $4.1, n \geq 12$.

Note that the connected 5-regular $K_{3}-\gamma$-excellent graph depicted in Fig. 2 has order 12.

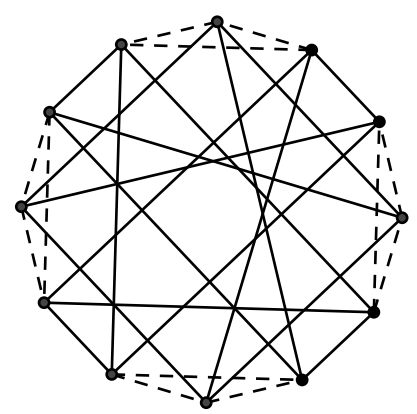

Figure 2: A 5-regular $K_{3}$ - $\gamma$-excellent connected graph on 12 vertices.

Now we concentrate on graphs having cut-vertices.

Let $G_{1}, G_{2}, \ldots, G_{k}$ be pairwise disjoint connected graphs of order at least 2 and $v_{i} \in V\left(G_{i}\right), i=1,2, \ldots, k$. Then the coalescence $\left(G_{1} \cdot G_{2} \cdot \ldots \cdot G_{k}\right)\left(v_{1}, v_{2}, \ldots, v_{k}: v\right)$ of $G_{1}, G_{2}, \ldots, G_{k}$ via $v_{1}, v_{2}, \ldots, v_{k}$, is the graph obtained from the union of $G_{1}, G_{2}, \ldots, G_{k}$ by identifying $v_{1}, v_{2}, \ldots, v_{k}$ in a vertex labeled $v$. If for graphs $G_{1}, G_{2}, \ldots, G_{k}$ is fulfilled $V\left(G_{i}\right) \cap V\left(G_{j}\right)=\{x\}$ when $i, j=1,2, \ldots, k$ and $i \neq j$, then the coalescence $\left(G_{1} \cdot G_{2} \cdot \ldots \cdot G_{k}\right)(x)$ of $G_{1}, G_{2}, \ldots, G_{k}$ via $x$ is the union of $G_{1}, G_{2}, \ldots, G_{k}$.

Define $V^{-}(G)=\{x \in V(G) \mid \gamma(G-x)<\gamma(G)\}$ and $V^{=}(G)=\{x \in V(G) \mid \gamma(G-x)=\gamma(G)\}$. It is well known that $V^{-}(G)=\{x \in V(G) \mid \gamma(G-x)+1=\gamma(G)\}$. To continue we need the following result:

Lemma 4.1. [2] Let $G=(F \cdot H)(x)$. Then $x \in V^{-}(G)$ if and only if $x \in V^{-}(F) \cap V^{-}(H)$. Furthermore, if $x \in V^{-}(G)$, then $\gamma(G)=\gamma(F)+\gamma(H)-1$.

Theorem 4.3. Let $G=\left(G_{1} \cdot G_{2} \cdot \ldots \cdot G_{k}\right)(x), x \in V^{-}(G)$ and $G_{i}$ is $H$ - $\gamma$-exellent, $i=1,2, \ldots, k$, where $H$ is connected and has no cut-vertex. Then $G$ is also $H$ - $\gamma$-excellent.

Proof. Using induction on $k$ we easily obtain from Lemma 4.1 that $\{x\}=V^{-}\left(G_{1}\right) \cap V^{-}\left(G_{2}\right) \cap \ldots \cap V^{-}\left(G_{k}\right)$ and $\gamma(G)=$ $\gamma\left(G_{1}\right)+\gamma\left(G_{2}\right)+\ldots+\gamma\left(G_{k}\right)-k+1$. Consider any induced subgraph $R$ of $G$, which is isomorphic to $H$. Since $H$ is connected and without cut-vertices, $R$ is an induced subgraph of some $G_{i}$, say without loss of generality, $i=1$. Then there is a $\gamma$-set $D_{1}$ of $G_{1}$ for which $R$ is an induced subgraph of $\left\langle D_{1}\right\rangle$. Let $D_{i}$ be a $\gamma$-set of $G_{i}-x, i=2,3, \ldots, k$. Since $x \in V^{-}\left(G_{i}\right)$, $\left|D_{i}\right|=\gamma\left(G_{i}\right)-1$. Then $D=\cup_{i=1}^{k} D_{i}$ is a $\gamma$-set of $G$ and $R$ is an induced subgraph of $\langle D\rangle$.

Define a vertex labeling of a tree $T$ as a function $S: V(T) \rightarrow\{0,1\}$. A labeled tree $T$ is denoted by a pair $(T, S)$. Let $\mathbf{0}_{T}$ and $\mathbf{1}_{T}$ be the sets of vertices assigned the values 0 and 1, respectively. In a labeled 1-corona tree $T$ of order at least four all its leaves are in $\mathbf{0}_{T}$ and all its support vertices form $\mathbf{1}_{T}$.

Let $\mathscr{T}$ be the family of labeled trees $(T, S)$ that can be obtained from a sequence of labeled trees $\tau:\left(T^{1}, S^{1}\right), \ldots,\left(T^{j}, S^{j}\right)$, $(j \geq 1)$, such that $\left(T^{1}, S^{1}\right)$ is a labeled 1-corona tree of order at least four and $(T, S)=\left(T^{j}, S^{j}\right)$, and, if $j \geq 2,\left(T^{i+1}, S^{i+1}\right)$ can be obtained recursively from $\left(T^{i}, S^{i}\right)$ by the following operation (a visual example of this operation is given in Figure $3)$ :

Operation $\boldsymbol{O}$. The labeled tree $\left(T^{i+1}, S^{i+1}\right)$ is obtained from vertex disjoint $\left(T^{i}, S^{i}\right)$ and a labeled 1-corona tree $G_{i}$ in such a way that $T^{i+1}=\left(T^{i} \cdot G_{i}\right)(u, v: u)$, where (a) $u \in \mathbf{0}_{T^{i}}, v \in \mathbf{0}_{G_{i}}$ and $u \in \mathbf{0}_{T^{i+1}}$, and (b) $\mathbf{0}_{T^{i+1}}=\mathbf{0}_{T^{i}} \cup \mathbf{0}_{G_{i}}-\{v\}$ and $\mathbf{1}_{T^{i+1}}=\mathbf{1}_{T^{i}} \cup \mathbf{1}_{G_{i}}$.

Now we are in a position to present a (reformulated) constructive characterization of $\gamma$-excellent trees.

Theorem 4.4. [17] For any tree $T$ of order at least four the following are equivalent:

(i) $T$ is $\gamma$-excellent.

(ii) There is labeling $S: V(T) \rightarrow\{0,1\}$ such that $(T, S)$ is in $\mathscr{T}$.

Moreover, if $(T, S)$ is in $\mathscr{T}$, then $\boldsymbol{0}_{T}=V^{-}(T), \boldsymbol{0}_{T}$ is a $\gamma$-set of $T$ and $\mathbf{1}_{T}=V^{=}(T)$. In particular, all leaves of $T$ are in $V^{-}(T)$. 

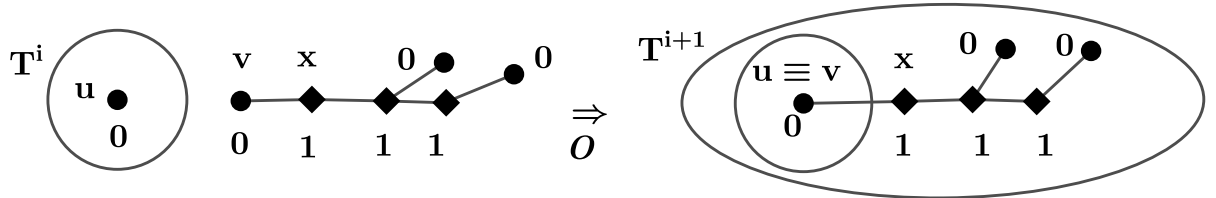

Figure 3: An example of Operation $\boldsymbol{O}$.

Another constructive characterization of the $\gamma$-excellent trees can be found in [3]. To prove our last result we need the following lemma.

Lemma 4.2. Let $G$ be a connected graph and $x \in V^{-}(G)$.

(i) If $x y$ is a bridge in $G$, then no $\gamma$-set of $G$ contains both $x$ and $y$.

(ii) If $x y$ and $x z$ are bridges in $G$, then no $\gamma$-set of $G$ contains both $y$ and $z$.

Proof. (i) Clearly, we can consider $G$ as a coalescence $(F \cdot H)(x)$, where without loss of generality, $y \in V(F)$ and $x$ is a leaf of $F$. Suppose $D$ is a $\gamma$-set of $G$ and $x, y \in D$. Then $D \cap V(H)$ and $D \cap V(F)$ are dominating sets of $H$ and $F$, respectively. Moreover, since $x$ is a leaf in $F, D \cap V(F)$ is not a $\gamma$-set of $F$. Hence $|D|=|D \cap V(H)|+|D \cap V(F)|-1 \geq \gamma(H)+(\gamma(F)+1)-1$, a contradiction with Lemma 4.1 .

(ii) Let as in (i), $G=(F \cdot H)(x), y \in V(F)$ and $x$ is a leaf of $F$. Hence $z \in V(H)$. Let $D$ be a $\gamma$-set of $G$ and $y, z \in D$. By (i), $x \notin D$ and then $D \cap V(H)$ and $D \cap V(F)$ are dominating sets of $H$ and $F$, respectively. This implies $|D|=|D \cap V(H)|+$ $|D \cap V(F)| \geq \gamma(H)+\gamma(F)$, a contradiction with Lemma 4.1.

Theorem 4.5. Let $T$ be a $\gamma$-excellent tree of order at least four.

(a) If $T$ has a cut-vertex belonging to $V^{-}(T)$, then $T\langle\gamma\rangle=\left\{K_{1}\right\}$.

(b) If no cut-vertex of $T$ is in $V^{-}(T)$, then $T$ is a 1-corona tree and $T\langle\gamma\rangle=\left\{\overline{K_{1}}, \ldots, \overline{K_{r}}\right\}$, where $2 r=|V(T)|$.

Proof. Suppose $H \in T\langle\gamma\rangle$ and $H$ is not edgeless. Let $D$ be a $\gamma$-set of $T$ and $R \simeq H$ be an induced subgraph of $\langle D\rangle$. Choose arbitrarily an edge $x y$ of $R$. Clearly both $x$ and $y$ are not leaves and by Lemma 4.2, neither $x$ nor $y$ is a cut-vertex belonging to $V^{-}(T)$. Hence $x, y \in V^{=}(T)$, because of Theorem 4.4. Now we choose $x y$ so that $x$ is a leaf in $R$. By Theorem 4.4, a vertex $y$ has a neighbor $z \in V^{-}(T)$. Lemma 4.2 now implies $N[z] \cap D=\{y\}$. But then the graph $R_{x}=\langle V(R-x) \cup\{z\}\rangle$ is isomorphic to $R$. Since $z \in V^{-}(T)$ and $y z \in E(T)$, Lemma 4.2 shows that no $\gamma$-set of $T$ contains both $y$ and $z$. Thus, we arrive to a contradiction.

Therefore, $T\langle\gamma\rangle$ contains only edgeless graphs. By Theorem $4.4 V^{-}(T)$ is a $\gamma$-set of $T$. Assume first that there is a cutvertex $x \in V^{-}(T)$. Then for any two neighbors $y$ and $z$ of $x$ the set $V_{1}=\left(V^{-}(T)-\{x\}\right) \cup\{y, z\}$ is independent of cardinality $\gamma(T)+1$. Suppose $T$ is $\overline{K_{r}}-\gamma$-excellent for some $r \geq 2$. Choose any cardinality $r$ subset $V_{1}$ of $\left(V^{-}(T)-\{x\}\right) \cup\{y, z\}$ that contains both $y$ and $z$. Now by Lemma 4.2, we conclude that no $\gamma$-set of $T$ has $V_{1}$ as a subset. Thus, $T\langle\gamma\rangle=\left\{K_{1}\right\}$.

Finally, let $V^{-}(T)$ contains only leaves. By Theorem 4.4, $T$ is a 1-corona tree. Clearly $\gamma(T)=i(T)=\beta_{0}(T)=r$ and then the required now follows by Proposition 2.1.

\section{Open problems and questions}

We conclude the paper by listing some interesting problems and directions for further research.

- For which ordered pairs $(r, s)$ there are $s$-regular $K_{r}$-excellent graphs of order $r(s-r+2)$ (see Theorem 4.2)? Find all 12-order 5-regular $K_{3}$ - $\gamma$-excellent graphs.

- Characterize/describe all graphs $F$ such that there is no $F$ - $\mu$-excellent graph $G$ with $\mu(G)=|V(F)|$ (see Observation 1.1). Recall that there is no $P_{3}$ - $\gamma$-excellent graph $G$ with $\gamma(G)=3$ (Theorem 2.1).

- Let $b$ be a positive integer. Denote by $\mathscr{A}(\mu, b)$ the class of all $\mu$-excellent connected graphs $G$ for which $\mu(G)=b$ and $|G\langle\mu\rangle|$ is maximum. It might be interesting for the reader to investigate these classes at least when $b$ is small. Note that we already know that $\mathscr{A}(\gamma, 1)$ consists of all complete graphs, and all connected graphs obtained from $K_{2 n}$, $n \geq 2$, by removing a perfect matching form $\mathscr{A}(\gamma, 2)$ (Example 2.1). In addition, by Example 2.4 we have $\gamma\left(K_{3} \square K_{3}\right)=3$, $K_{3} \square K_{3}\langle\gamma\rangle=\left\{K_{1}, \overline{K_{2}}, K_{2}, K_{1} \cup K_{2}, \overline{K_{3}}, K_{3}\right\}$ and by Theorem 2.1 we know that there is no $P_{3}$ - $\gamma$-excellent graph $G$ with $\gamma(G)=3$. Thus, $K_{3} \square K_{3}$ belongs to $\mathscr{A}(\gamma, 3)$ and $\left|K_{3} \square K_{3}\langle\gamma\rangle\right|=6$. Find $\mathscr{A}(\gamma, 3)$. 
- Find $T\langle\mu\rangle$ for each $\mu$-excellent tree $T$, where $\mu \in\left\{i, \gamma_{t}, \gamma_{R}\right\}$ and $\gamma_{R}$ stand for the Roman domination number (see [9], [10] and [18], respectively).

- Find graphs $H$ such that each induced subgraph of $K_{p} \square H$ which is isomorphic to $H$ has as a vertex set some $H$-layer (see Theorem 2.3).

- Characterize/describe all connected $\overline{K_{2}}-\gamma$-excellent graphs $G$ with $\gamma(G)=2$.

\section{Acknowledgment}

The author express his sincere thanks to the anonymous referees for their meticulous and thorough reading of the paper that greatly improved its exposition.

\section{References}

[1] C. Berge, Some common properties for regularizable graphs, edge-critical graphs, and b-graphs, In: N. Saito, T. Nishizeki (Eds.), Graph Theory and Algorithms, Lecture Notes in Computer Science Vol. 108, Springer, Berlin, 1981, pp. 108-123.

[2] R. C. Brigham, P. Z. Chinn, R. D. Dutton, Vertex domination-critical graphs, Networks 18 (1988) 173-179.

[3] T. Burton, D. P. Sumner, $\gamma$-Excellent, critically dominated, end-dominated, and dot-critical trees are equivalent, Discrete Math. 307 (2007) $683-693$.

[4] N. Dean, J. Zito, Well-covered graphs and extendability, Discrete Math. 126 (1994) 67-80.

[5] M. El-Zahar, C. M. Pareek, Domination number of products of graphs, Ars Combin. 31 (1991) 223-227.

[6] G. Fricke, T. Haynes, S. Hedetniemi, S. Hedetniemi, R. Laskar, Excellent trees, Bull. Inst. Combin. Appl. 34(2002) 27-38.

[7] P. J. P. Grobler, Critical Concepts in Domination, Independence and Irredundance of Graphs, PhD thesis, University of South Africa, Pretoria, 1998.

[8] T. W. Haynes, S. T. Hedetniemi, P. J. Slater, Fundamentals of Domination in Graphs, Marcel Dekker, New York, 1998.

[9] T. W. Haynes, M. A. Henning, A characterization of $i$-excellent trees, Discrete Math. 248 (2002) 69-77.

[10] M. A. Henning, Total domination excellent trees, Discrete Math. 263 (2003) 93-104.

[11] S. R. Jayaram, Minimal dominating sets of cardinality two in graphs, Indian J. Pure Appl. Math. 28 (1997) 43-46.

[12] M. Meringer, Fast generation of regular graphs and construction of cages, J. Graph Theory 30 (1999) 137-146.

[13] M. Meringer, www.mathe2.uni-bayreuth.de/markus/reggraphs.html\#CRG

[14] C. M. Mynhardt, H. C. Stwart, E. Ungerer, Excellent trees and secure domination, Utilitas Math. 67 (2005) $255-267$.

[15] M. D. Plummer, Some covering concepts in graphs, J. Combin. Theory 8 (1970) 91-98.

[16] B. Reed, Paths, stars and the number three, Combin. Probab. Comput. 5 (1996) 267-276

[17] V. Samodivkin, Domination in graphs, God. Univ. Arkhit. Stroit. Geod. Sofiya Svitk II Mat. Mekh. 39 (1996-1997) 111-135.

[18] V. Samodivkin, Roman domination excellent graphs: trees, Commun. Comb. Optim. 3 (2018) 1-24.

[19] Y. Sohn, X. Yuan, Domination in graphs of minimum degree four, J. Korean Math. Soc. 46 (2009) 759-773.

[20] N. Sridharan, M. Yamuna, Very excellent graphs and rigid very excellent graphs, AKCE J. Graphs. Combin. 4 (2007) 211-221.

[21] D. P. Sumner, P. Blitch, Domination critical graphs, J. Combin. Theory Ser. B 34 (1983) 65-76.

[22] H. M. Xing, L. Sun, X. G. Chen, Domination in graphs of minimum degree five, Graphs Combin. 22 (2006) 127-143.

[23] M. Yamuna, N. Sridharan, Just excellent graphs, Int. J. Engin. Sci. Adv. Comput. Bio-Technol. 1 (2010) $129-136$. 